\title{
Criminologie
}

\section{La commission de réforme du droit du Canada : un bilan succinct}

\section{Jacques Fortin}

Volume 15, numéro 1, 1982

Droit et justice

URI : https://id.erudit.org/iderudit/017152ar

DOI : https://doi.org/10.7202/017152ar

Aller au sommaire du numéro

Éditeur(s)

Les Presses de l'Université de Montréal

ISSN

0316-0041 (imprimé)

1492-1367 (numérique)

Découvrir la revue

Citer cet article

Fortin, J. (1982). La commission de réforme du droit du Canada : un bilan succinct. Criminologie, 15(1), 105-112. https://doi.org/10.7202/017152ar d'utilisation que vous pouvez consulter en ligne.

https://apropos.erudit.org/fr/usagers/politique-dutilisation/ 
La Commission de réforme du droit du Canada a maintenant dix ans ${ }^{1}$. Pendant ces dix années, elle s'est manifestée, au moins dans le monde juridique du pays, par la publication de nombreux rapports, documents de travail et documents d'étude. Son mandat spécifique consiste "à revoir et à étudier, d'une façon continuelle et systématique, les lois et règles de droit qui constituent le droit du Canada, en vue de faire des propositions pour les améliorer, moderniser et réformer $"$.

Tâche énorme, dont les difficultés n'ont pas échappé au législateur puisqu'il a jugé nécessaire de faire de la Commission de réforme du droit une institution permanente. Mais au bout de dix ans, on peut tout de même se demander où en est la Commission dans l'accomplissement de son mandat.

Pour répondre à cette question, on est naturellement porté à consulter les recueils de lois de manière à déterminer le nombre et l'importance des recommandations de la Commission qui, pendant les dix dernières années, se sont traduites dans des textes de loi. La réponse à cette question est simple : rien. Elle est aussi inquiétante pour la validité du processus de réforme du droit, car, on s'en doute bien, la Commission n'a pas été inactive pendant ces dix années. Loin de là. Elle a déposé devant le Parlement canadien pas moins de quatorze rapports comportant des projets de réforme législative touchant au droit pénal, au droit de la preuve, au droit de la famille et au droit administratif. Une foule de projets de loi incorporant des recommandations mises de l'avant par la Commission ont été soumis au Parlement, mais aucun n'a encore été adopté. Dans certains domaines, celui de la famille par exemple, cela peut s'expliquer par le fait que la réforme exige une action concertée entre le Parlement fédéral et les législatures provinciales, et on peut souhaiter qu'à leur égard ce qui semble être une inertie gouvernementale ne soit qu'une lenteur administrative. Dans d'autres, celui du droit pénal par exemple, l'inaction législative trouve son explication dans certains

* Professeur à la Faculté de droit de l'Université de Montréal.

1. La loi instituant la Commission de réforme du droit, sanctionnée le 26 juin 1970 , est entrée en vigueur le 12 juin 1971. 
facteurs que nous nous proposons d'analyser brièvement en faisant le bilan des travaux de la Commission. Nous verrons d'ailleurs que la réforme du droit pénal n'est pas compromise puisque les mesures susceptibles d'en accélérer la réalisation sont maintenant en place.

La Commission a été consciente, dès le début de ses travaux, des exigences de son mandat qui lui posait le défi de repenser le système de droit en fonction des besoins de la société canadienne moderne. Il ne s'agissait donc pas d'une simple révision des lois, mais bien d'une réforme du droit.

La Commission doit bien sûr supprimer les anachronismes et les anomalies du droit. Mais elle doit aussi faire un travail en profondeur de manière à « développer de nouvelles méthodes et de nouveaux concepts de droit correspondant à l'évolution des besoins de la société canadienne moderne et des individus qui la compo$\operatorname{sent}^{2}$ ").

Aussi, la Commission a-t-elle tenté, comme elle s'en explique dans son deuxième rapport annuel ${ }^{3}$, de donner à ses travaux les deux dimensions qu'elle jugeait essentielles à l'accomplissement de son mandat : d'une part, la recherche d'une théorie fondamentale qui lui donnerait une grille d'analyse des objectifs de la réforme; ensuite, l'étude du droit actuel, non seulement dans son contenu formel, qui suppose la consultation des ouvrages, des lois et de la jurisprudence, mais aussi dans sa dimension concrète qui exige le support de recherches empiriques.

De plus, acceptant l'idée que c'est le concensus social qui donne à la loi sa légitimité, la Commission a également insisté sur l'importance en profondeur de consultations publiques tant sur l'orientation de son programme de recherches que sur le contenu de ses recommandations. D'où cette distinction qu'elle a faite dès le début de ses travaux sntre trois sortes de publications : d'abord, un document d'étude qui a pour objet de fournir au lecteur les données d'un

2. Art. 11 de la Loi sur la Commission de réforme du droit.

3. Information Canada, Ottawa (1972). La Commission était à ce moment présidèe par le juge E. Patrick Hart de la Cour suprême d'Ontario. Le juge Antonio Lamer, nommé depuis à la Cour d'appel du Québec et ensuite à la Cour suprême du Canada, était vice-président. Il était alors juge de la Cour supérieure du Québec. Les commissaires étaient : Martin L. Friedland, professeur de droit à l'Université de Toronto, qui a démissionné après quelques mois pour devenir doyen de la Faculté de droit de l'Université de Toronto. Il a été remplacé par: Dr. Hans Mohr, professeur de criminologie à Osgoode Hall; William F. Ryan. professeur de droit à l'Université du Nouveau-Brunswick, nommé depuis juge à la Cour fédèrale, division d'appel; Claire Barrette-Joncas, commissaire à temps partiel, maintenant juge de la Cour supérieure du Québec; John D. McAlpine, également commissaire à temps partiel, membre du Barreau de la Colombie Britannique. 
problème, ensuite un document de travail où la Commission trace à titre provisoire les orientations d'une réforme. Ces deux documents doivent en principe avoir une large diffusion publique par le truchement d'organismes publics et privés de manière à informer les intéressés et à susciter leurs réactions. La Commission se ménageait aussi le temps de tenir des séances de consultations avec les représentants des institutions ou des organismes intéressés à ses travaux. Enfin, la troisième étape est celle du rapport où la Commission recommande au Parlement l'adoption d'une réforme.

La Commission a, jusqu'à maintenant, publié sept rapports touchant à divers aspects du droit pénal. Dès 1975, elle a recommandé au Parlement l'adoption d'un Code de la preuve devant remplacer l'actuelle Loi de la preuve qui date de $1892^{4}$. Dans la même année, elle a fait des recommandations touchant le désordre mental dans le processus pénal ${ }^{5}$. En 1976, elle a livré, dans un rapport intitulé "Notre droit pénal " ", la philosophie du droit pénal qui devrait, à son avis, guider l'action du législateur. L'année 1978 a vu la parution d'un rapport sur la procédure pénale?

La Commission a ensuite abordé le domaine des infractions en publiant deux rapports, l'un sur les infractions sexuelles ${ }^{8}$, l'autre sur le vol et la fraude 9 . Elle a également publié de nombreux documents de travail portant entre autres sur la stérilisation ${ }^{10}$, le traitement médical et le droit criminel ${ }^{11}$, le jury en droit pénal ${ }^{12}$, l'outrage au tribunal ${ }^{13}$, les critères de détermination de la mort ${ }^{14}$, etc.

Parmi tous les rapports, celui intitulé "Notre droit pénal " prend une importance particulière du fait qu'on y trouve, comme le dit la Commission dans son cinquième rapport annuel, "une réflexion globale sur le droit pénal ». Il est le résultat de trois années de

4. La preuve, le 19 décembre 1975, Commission de réforme du droit, No. cat. J31-15/1975.

5. Principes directeurs des sentences et mesures non sentencielles dans le processus pénal, le 6 février 1976, No. cat. J31-16/1975.

6. Notre droit pénal, le 25 mars 1975, Commission de réforme du droit, No. cat. J31-19/1976. Ce rapport est la synthèse de nombreux documents de travail : La déjudiciarisation, janvier 1975, No. cat. J32-1/7-1974; Les confins du droit pénal, juin 1975, No. cat. J32-1/10-1975.

7. Procédure pénale, 1 ere partie, amendements divers, le 23 février 1978.

8. Le 29 novembre 1978, base sur le document de travail $n^{\circ} 22$.

9 . Le 16 mars 1979 , base sur le document de travail $n^{\circ} 19$.

10. Document de travail $\mathrm{n}^{\circ} 24$.

11. Document de travail $\mathrm{n}^{\circ} 26$.

12. Document de travail $\mathrm{n}^{\circ} 27$.

13. Document de travail $\mathrm{n}^{\circ} 20$.

14. Document de travail $n^{\circ} 23$. On trouvera la liste des rapports et des documents de travail dans les rapports annuels de la Commission. 
recherches, de consultations et de réflexion sur les objectifs et la fonction du droit pénal ${ }^{15}$. Il énonce en effet les principes qui, selon la Commission, devraient orienter la réforme du droit pénal. Si celui-ci a pour fonction; comme nous le rapporte la Commission, « de réaffirmer et d'appuyer les valeurs sociales fondamentales », encore faut-il ne l'invoquer qu'à l'encontre des conduites vraiment répréhensibles qui supposent une culpabilité chez leurs auteurs ${ }^{16}$ et justifient ainsi le recours à l'emprisonnement ${ }^{17}$.

Le rapport esquisse en outre une réorganisation complète du Code criminel fondée sur la distinction entre le "crime véritable" et l' " infraction réglementaire 18 ", ainsi qu'un nouveau style d'écriture législative axé sur la simplicitél ${ }^{9}$. On voit ainsi qu'en cinq années la Commission a mis au monde sa philosophie et son plan de réforme du droit pénal et recommandé au législateur l'adoption de réformes concrètes de certaines parties du droit criminel, tout en poursuivant son objectif d'accomplir une réforme complète du Code criminel.

Sur le plan de la philosophie pénale, la Commission a recommandé au Parlement, entre autres mesures : 1. d'user de modération dans la définition des crimes, et réserver pour le Code criminel la définition des crimes véritables et insérer dans les lois particulières les infractions réglementaires; 2 . de repenser l'arsenal des peines de manière à appliquer l'emprisonnement uniquement aux crimes véritables; 3 . de réduire la durée des peines, sauf dans les cas où l'emprisonnement de longue durée est nécessaire à la neutralisation du coupable dangereux; 4 . d'éliminer la responsabilité pénald sans faute et de la remplacer par une responsabilité fondée sur la négligence; 5 . de modifier la procédure préalable au procès par l'implantation d'un système de communication de la preuve; 6 . de réorganiser

15. La méthodologie de cette recherche est exposée dans le deuxième rapport annuel (1972-73), $\mathrm{n}^{\circ}$ cat. J31-1973.

16. Voir sur le sujet la notion de blâme. Document de travail $n^{\circ} 2$, février 1974, $\mathrm{n}^{\circ}$ cat. J32-1/2-1974, et Études sur la responsabilité stricte, $\mathrm{n}^{\circ}$ cat. J32-4/11974.

17. Principes directeurs des sentences et mesures non sentencielles dans le processus pénal, Rapport, février 1976, $\mathrm{n}^{\circ}$ cat. J31-19/1976, et le document de travail $n^{\circ} 3$. Les principes de la détermination de la peine et du prononcé de la sentence (1974), $\mathrm{n}^{\mathrm{O}}$ cat. J32-1/3-1974. Voir aussi Document de travail 11, Emprisonnementlibération (1975), $\mathrm{n}^{\mathrm{O}}$ cat. J32-1/11-1975, et les documents d'études suivants : Études sur l'emprisonnement, juillet 1976, $\mathrm{n}^{\circ}$ cat. J32-4/9-1975; Liberté au compte-gouttes, octobre 1976, $\mathrm{n}^{\circ}$ cat. J32-4/12-1976; La gravité du châtiment, mai 1976, $\mathrm{n}^{\circ}$ cat. J32$4 / 10-1975$.

18. Sur cette question, voir études sur la responsabilité stricte, note 16 supra.

19. Voir le document d'études: "Pour une codification du droit pénal canadien ", avril 1976, no cat. J3!-261976. 
les mesures non sentencielles destinées aux prévenus inaptes, en raison d'une déficience mentale, à subir leur procès. Toutes ces recommandations sont appuyées par la démonstration de mesures concrètes susceptibles d'en favoriser l'implantation.

D'une manière plus ponctuelle, la Commission a également recommandé au Parlement l'adoption 1. d'un Code de la preuve, 2. d'une nouvelle codification des infractions sexuelles, et 3 . des crimes contre la propriété.

Mais il est bientôt devenu évident que la Commission aurait beau publier rapports sur rapports, recommandations sur recommandations, la réforme du droit pénal risquait de rester dans les tiroirs. La rareté des modifications législatives auxquelles avaient donné lieu ses propositions permettait de le penser ${ }^{20}$. Il y avait certes des preuves tangibles de son influence sur la réforme du droit dans les secteurs judiciaire et administratif ${ }^{21}$, mais sur le plan législatif, elle ne pouvait que déplorer qu'aucun des nombreux projets de lois fondés directement ou indirectement sur ses travaux n'avait reçu la sanction du Parlement ${ }^{22}$.

Il est difficile d'analyser d'une manière précise les causes de cette situation. Ce n'est certes pas la volonté politique de réformer le droit qui fait défaut. La création d'une commission de réforme du droit témoigne assez de cette volonté ${ }^{23}$. Mais on peut dégager deux

20. 7. rapport annuel, p. 4 à 7.

21. Les 7.8 et 9: rapports annuels font un relevé des références aux travaux de la Commission que l'on trouve dans les arrêts. Ils montrent que la Commission exerce une influence certaine sur l'orientation des décisions judiciaires. Pour n'en donner ici qu'un exemple, mentionnons l'arrêt R. c. Sault Ste-Marie (1978) 2 R.C.S. 1299, dans lequel la Cour suprême du Canada adopte, en la mentionnant explicitement, la recommandation de la Commission de réforme du droit de substituer à la responsabilité absolue une responsabilité fondée sur la négligence en matières d'infractions réglementaires. L'influence sur le secteur administratif est illustrée par les réactions de la Commission d'assurance-chômage aux recommandations contenues dans une étude de sa procédure administrative. Dans son 7: rapport annuel, la Commission de réforme du droit cite une letıre du président de la C.A.C. où il est dit que la C.A.C. avait déjà mis en ceuvre 20 recommandations parmi les 68 contenues dans l'étude et qu'elle comptait en appliquer 31 autres.

22. De nombreuses recommandations de la Commission ont été incluses dans des projets de lois. La Commission en fait une analyse détaillée dans les $7 e$, $8 \mathrm{c}$ et $9 \mathrm{c}$ rapports annuels. Nous mentionnons ici, à titre d'exemple, le Bill omnibus de 1978, (C-51) concernant les dispositions relatives au dédommagement et à la restitution, aux motifs de la sentence et de sa durée, aux ordonnances de service communautaire, directement inspirées par le rapport de la Commission sur les sentences et les mesures non sentencielles (1976). Le même projet de loi portait aussi sur la procédure pénale préconisée dans le rapport de la Commission sur la procédure (1978). Outre les projets de lois présentés par le ministère de la Justice, plusieurs projets de lois présentés par les députés ont aussi repris plusieurs recommandations de la Commission.

23. Dans le 7e rapport annuel, la Commission se dit certaine de l'existence d'une volonté politique de réforme du droit tant dans la population que chez les membres du Parlement et du Sénat. Elle mentionne toutefois qu'il existe un courant anti-réformiste. 
facteurs de ralentissement du processus : le premier tient aux structures de l'appareil gouvernemental fédéral; le second, du reste relié au premier, tient au fait que la réforme du droit se répercute sur l'administration de la justice et elle exige ainsi la participation des provinces.

La Commission de réforme du droit, quoique placée sous la responsabilité du ministre de la Justice du Canada, est un organisme indépendant du ministère de la Justice. La Commission achemine ses rapports au Parlement par l'entremise du ministre de la Justice qui doit en faire le dépôt devant le Parlement ${ }^{24}$. La loi lui reconnaît toutefois le droit de déposer devant le Parlement ses commentaires sur les recommandations de la Commission ${ }^{25}$. Cette structure, dont le but fort louable d'ailleurs, était d'assurer à la Commission l'indépendance d'esprit nécessaire à la conception d'une réforme du droit, comporte le désavantage de dissocier le ministère de la Justice, qui est responsable devant le Parlement des projets de loi qui y sont présentés, des travaux de continuité entre les recommandations de la Commission et leur mise en oeuvre législative par le ministère de la Justice.

Le sort du Code de la preuve illustre ce phénomène. La Commission l'a préparé à l'intention du Parlement en se basant sur des consultations intensives auprès des juges et des avocats, consultations alimentées par des recherches faites en bibliothèque ou sur le terrain, qui avaient toutes été publiées sous la forme de documents d'étude. Avant de recommander au Parlement l'adoption du code de la preuve, sous sa forme originale ou sous une forme modifiée, et donc d'y engager sa responsabilité, le ministère de la Justice s'est vu dans l'obligation de reprendre tout le processus à zéro : embauche de chercheurs et de consultants, mise sur pied de groupes de travail et de consultation, etc. Tant et si bien qu'il n'y a pas encore devant le Parlement de projet de loi sur la codification totale ou partielle de la Loi de la preuve. Le projet de réforme des infractions sexuelles est peut-être une illustration encore plus frappante du défaut de continuité entre le Ministère et la Commission. Lorsque le

24. La Commission est tenue de soumettre à l'approbation du Ministre de la justice son programme de recherches. Celui-ci peut requérir de la Commission qu'elle accorde une priorité à un sujet particulier. Quant au reste. la Commission est autorisée par la loi à entreprendre les études qu'elle juge nécessaires à l'accomplissement de son mandat.

25. La Commission soumet ses rapports au ministre de la Justice. Celui-ci doit, dans les quinze jours de la réception d'un rapport, le déposer au Parlement, ou, si le Parlement ne siège pas à ce moment-là. le déposer dans les quinze jours où il recommence à siéger. 
ministre de la Justice a déposé le Bill C-52 devant le Parlement, il a mentionné la publication imminente d'un document de travail de la Commission portant sur ce secteur du droit. Il était donc évident que le ministère de la Justice et la Commission de réforme du droit avaient travaillé séparément sur le même sujet, l'un prenant l'autre de vitesse. Mentionnons toutefois que le Bill C-52 n'a pas encore été adopté, pas plus du reste que le rapport de la Commission. Ces deux exemples, parmi d'autres, marquent un manque de complémentarité entre la Commission et le ministère de la Justice.

Le deuxième facteur de ralentissement de la mise en ouvre des recommandations de la Commission tient au rôle que les administrateurs de la justice pénale doivent jouer dans la réforme du droit. Celle-ci peut en effet, entraîner des modifications de l'infrastructure nécessaire à l'administration de la justice (que l'on pense, par exemple, aux étapes préalables au procès), et de ce fait, entrâ̂ner des coûts additionnels se répercutant sur les budgets provinciaux. Certes, la Commission a toujours été consciente de la nécessité de consulter les organismes chargés de l'application des lois de manière à ne pas perdre de vue les dimensions concrètes des problèmes. Mais ces consultations, n'étant ni systématiques ni institutionnalisées, semblaient avoir peu de poids aux yeux des ministères de la Justice fédéral et provincial.

Pour pallier à toutes ces difficultés, la Commission a suggéré à plusieurs reprises que ses rapports reçoivent au moins un examen parlementaire rapide qui lui permette, d'une part, de justifier ses recommandations devant le Parlement, et d'autre part, de planifier ses travaux à venir ${ }^{26}$.

Ces suggestions n'ont pas été retenues comme telles mais elles semblent avoir eu un résultat qui est loin d'être négligeable. En effet, les ministères fédéral et provinciaux de la Justice ont, de concert avec la Commission de réforme du droit, mis sur pied une structure administrative susceptible d'assurer une coordination efficace de leurs travaux et de leurs responsabilités respectives. Sans entrer dans tous les détails de cette structure 27 , il faut en mentionner deux aspects importants. D'abord, l'objectif est d'accélérer le processus de la réforme du droit pénal que l'on espère compléter dans une période de cinq années. Ensuite, la Commission continuant à jouer son rôle dans la conception et l'impulsion des réformes, le ministère

26. $8^{\mathrm{e}}$ rapport annuel. p. 6 et 7. 15 à 17.

27. Ce programme est exposé dans le $9^{\mathrm{e}}$ rapport annuel de la Commission, p. 
de la Justice entend également jouer le sien au niveau de la mise en œuvre des recommandations de la Commission. La continuité entre les travaux de la Commission (Phase I) et ceux du Ministère (Phase II) est assurée par des mécanismes prévoyant la consultation des représentants des ministères provinciaux de la Justice, tant dans la Phase I que dans la Phase II. Il est également prévu qu'il y aura interaction entre les deux phases, le Ministère étant tenu au courant de l'orientation des travaux de la Commission, et celle-ci à son tour suivant de près la mise en œuvre de ses recommandations.

Cette nouvelle structure fonctionne depuis moins d'un an. C'est ainsi que tous les rapports que la Commission a présentés au Parlement au cours des dernières années sont présentement à l'étude au ministère de la Justice qui doit déterminer les conditions de leur mise en œuvre. Entre-temps, la Commission poursuit son programme de réforme, à cette différence près qu'elle consulte maintenant d'une manière systématique et continue les représentants des ministères de la Justice. Il faut souligner, toutefois, que la Commission continue de consulter le Barreau, la magistrature, et les organismes qui peuvent apporter une contribution à l'orientation de ses recherches et de ses politiques de réforme.

Ce programme, dont l'objectif est l'adoption d'un Code criminel moderne avant cinq ans, porte tant sur les règles de fond du droit pénal (infractions, interprétation, responsabilité, moyens de défense et peines) que sur la procédure (pouvoirs et pratiques de la police, procédure préalable au procès, le procès, l'appel, etc.). Par exemple, cette année, la Commission a soumis à la consultation des représentants des ministères de la Justice et d'un groupe de juges, un projet de partie générale du Code criminel contenant les règles relatives à la responsabilité pénale et aux moyens de défense. Elle a aussi consulté les mêmes groupes sur l'orientation de la réforme des infractions contre la personne (voies de fait et homicide), ainsi que sur un projet de classification des infractions, sans parler de multiples consultations sur des questions de procédure (arrestation, perquisition, écoute électronique, etc.).

Il y a lieu de penser que la réforme du droit pénal canadien va s'accomplir à la suite de ce second souffle, du fait que les recommandations de la Commission auront un plus grand impact sur le Parlement en raison des consultations systématiques qu'elle aura menées auprès des décideurs du système pénal. 